\title{
Randomized Clinical Trial of Laparoscopic Versus Open Repair of the Perforated Peptic Ulcer: The LAMA Trial
}

\author{
Mariëtta J. O. E. Bertleff · Jens A. Halm • Willem A. Bemelman · \\ Arie C. van der Ham · Erwin van der Harst · Hok I. Oei · J. F. Smulders · \\ E. W. Steyerberg · Johan F. Lange
}

Published online: 9 May 2009

(c) The Author(s) 2009. This article is published with open access at Springerlink.com

\begin{abstract}
Background Laparoscopic surgery has become popular during the last decade, mainly because it is associated with fewer postoperative complications than the conventional open approach. It remains unclear, however, if this benefit is observed after laparoscopic correction of perforated peptic ulcer (PPU). The goal of the present study was to evaluate whether laparoscopic closure of a PPU is as safe as conventional open correction.

Methods The study was based on a randomized controlled trial in which nine medical centers from the Netherlands participated. A total of 109 patients with symptoms of PPU and evidence of air under the diaphragm were scheduled to receive a PPU repair. After exclusion of 8 patients during the operation, outcomes were analyzed for laparotomy $(n=49)$ and for the laparoscopic procedure $(n=52)$.

Results Operating time in the laparoscopy group was significantly longer than in the open group (75 min versus
\end{abstract}

\section{J. O. E. Bertleff ( $\square)$}

Department of Plastic and Reconstructive Surgery, Academic Hospital Maastricht, P. Debeyelaan 25, Maastricht 6229 HX,

The Netherlands

e-mail: doc.bert@orange.nl

J. A. Halm · J. F. Lange

Department of General Surgery, Erasmus MC, s-Gravendijkwal

230, Rotterdam 3015 GJ, The Netherlands

W. A. Bemelman

Department of General Surgery, AMC, Postbus 22660 ,

Amsterdam 1100 DD, The Netherlands

A. C. van der Ham

Department of General Surgery, SFG, Postbus 10900,

Rotterdam 3004 BA, The Netherlands
$50 \mathrm{~min}$ ). Differences regarding postoperative dosage of opiates and the visual analog scale (VAS) for pain scoring system were in favor of the laparoscopic procedure. The VAS score on postoperative days 1,3 , and 7 was significant lower $(P<0.05)$ in the laparoscopic group. Complications were equally distributed. Hospital stay was also comparable: 6.5 days in the laparoscopic group versus 8.0 days in the open group $(P=0.235)$.

Conclusions Laparoscopic repair of PPU is a safe procedure compared with open repair. The results considering postoperative pain favor the laparoscopic procedure.

\section{Introduction}

The incidence of perforated peptic ulcer (PPU) has declined over the past several years because of the introduction of anti-ulcer medication and Helicobacter eradication therapy

E. van der Harst

Department of General Surgery, MCRZ, Groene Hilledijk 315,

Rotterdam 3075 EA, The Netherlands

H. I. Oei

Department of General Surgery, RdGG, Reinier de Graafweg

3-11, Delft 2625 AD, The Netherlands

J. F. Smulders

Department of General Surgery, Catharina Hospital,

Michelangelolaan 2, Eindhoven 5623 EJ, The Netherlands

E. W. Steyerberg

Department of Public Health, Erasmus MC, s-Gravendijkwal 230, Rotterdam 3015 GJ, The Netherlands 
$[1,2]$. Nevertheless the incidence and mortality of PPU is $5-10 \%$. The mortality will increase up to $50 \%$ if the perforation exists for more than $24 \mathrm{~h}[3,4]$. There are several options for treatment of PPU, but the preferred treatment is surgery by upper abdominal laparotomy [5, 6].

Mouret et al. published the first results of laparoscopic repair in 1990 [7]. He concluded that it was a good method that probably reduced postoperative wound problems and adhesions. After the success of laparoscopic cholecystectomy and other laparoscopic procedures, it was thought that patients would have less pain and a shorter hospital stay after laparoscopic correction of PPU [8, 9]. Various studies have shown that laparoscopic suturing of the perforation is feasible, but there is still no proof of real benefits of laparoscopic correction [3, 6, 10-12]. Therefore we performed a multicenter randomized trial comparing open correction of PPU with laparoscopic repair.

\section{Methods}

\section{Participants}

Patients with symptoms of the clinical diagnosis of PPU were included in nine medical centers in the Netherlands participating in the LAMA (LAparoscopische MAagperforatie) trial between March 1999 and July 2005. Eligible patients were informed of the two surgical approaches and were invited to participate in the study. Exclusion criteria were the inability to read the Dutch language patient information booklet, inability to complete informed consent, prior upper abdominal surgery, and current pregnancy. The ethics committees of all participating institutions approved the trial.

\section{Randomization}

Surgeons contacted the study coordinator after the patients had provided informed consent and randomization took place by opening a sealed envelope. The envelope randomization was based on a computer-generated list provided by the trial statistician.

\section{Surgical procedure}

All patients received intravenous antibiotics prior to operation and were allocated for Helicobacter pylori eradication therapy according to established guidelines [13]. The open surgical procedure was performed through an upper abdominal midline incision. Closure of PPU was to be achieved by sutures alone or in combination with an omental patch. After repair of the defect cultures were drawn from the peritoneal fluid, after which the peritoneal cavity was lavaged. During lavage it was permissible to insufflate the stomach to test for leakage of the closed defect. No method was specified for closing the abdomen.

Laparoscopic repair was performed with the patient and the team set up in the "French" position. Trocars were placed at the umbilicus (video scope) and on the left and right midclavicular line above the level of the umbilicus (instruments). If necessary a fourth trocar was placed in the subxiphoid space for lavage or retraction of the liver. Surgeons were free to use either $0^{\circ}$ or $30^{\circ}$ video scopes for the procedure. The rest of the procedure was identical to that described above for open repair. No method was specified for closing the trocar incisions.

\section{Postoperative follow-up}

Postoperative pain was scored by means of a visual analog scale (VAS) for pain on days 1, 3, 7, and 28 ranging from 0 (no pain) to 10 (severe pain). In addition, the days during which opiates were used by the patients were registered. All complications, minor and major, were monitored. The treating surgeons determined time of discharge on the basis of physical well-being, tolerance of a normal diet, and ability to use the stairs. For this reason, this was an unblinded trial. Postoperative hospital stay without correction for time spent in hospital as a result of non-medical reasons (inadequate care at home) was calculated. Patients were invited to attend the outpatient clinic at 4 weeks, 6 months, and one year postoperatively. They were asked to complete forms related to pain and use of analgesics.

\section{Statistical analysis}

Data analysis was carried out according to the intention-totreat principle as established in the trial protocol. Data were collected in a database, and statistical analyses were performed with the Statistical Package for Social Sciences for Windows (SPSS 15.0, SPSS Inc., Chicago, IL). A researcher blinded to the nature of the procedures performed all data analyses. The primary outcome of the trial was duration of hospital stay. The power analysis was performed on basis of a reduction in hospital stay by 1.5 days (10-8.5 days from admission) in favor of the laparoscopically treated group using a $\beta$ of 0.80 and an $\alpha$ of 0.05 . This resulted in a trial size of 50 patients per group. The Pearson chi-squared test was used to compare categorical variables, and the Mann-Whitney U-test was used to compare continuous variables as we could not assume normal distribution because of the relatively small numbers. In Tables 1-6 medians and interquartile ranges (IQR) are reported. All data were analyzed according to the intention-to-treat principle; i.e., patients remained in their assigned group even if during the procedure the surgeon judged the patient not to be suitable for the technique 
Table 1 Baseline parameters

$I Q R$ interquartile range, difference between 25 th percentile and 75 th percentile; $B M I$ body mass index

ASA American Society of Anesthesiologists Association score

Table 2 Intraoperative findings

Table 3 Postoperative complications

\begin{tabular}{llll}
\hline & $\begin{array}{l}\text { Laparoscopic } \\
\text { repair } \\
n=52\end{array}$ & $\begin{array}{l}\text { Open } \\
\text { repair } \\
n=49\end{array}$ & $P$ value \\
\hline Pneumonia & 2 & 1 \\
Respiratory insufficiency & 1 & 3 \\
ARDS & 2 & 1 \\
Cardiac problems & 3 & 2 \\
Sepsis & 2 & 1 \\
Leakage at repair site & & \\
Abscess & & 3 \\
Ileus & & 1 \\
Fascial dehiscence & & 1 \\
Wound infection & & 3 \\
Urinary tract infection & & 2 \\
Incisional hernia & & 1 \\
Cerebrovascular accident & & 1 \\
Death & 2 & 4 \\
Total complications & 12 & 24 \\
Total of patients with & $9(18 \%)$ & $15(36 \%)$ \\
$\quad$ complications $\geq 1$ & & & 0.061 \\
\hline
\end{tabular}

Table 4 Duration of hospital stay, nasogastric decompression

\begin{tabular}{clll}
\hline & $\begin{array}{l}\text { Laparoscopic } \\
\text { repair } \\
n=52\end{array}$ & $\begin{array}{l}\text { Open } \\
\text { repair } \\
n=49\end{array}$ & $P$ value \\
\hline $\begin{array}{c}\text { Median hospital } \\
\text { stay (days) }+\end{array}$ & $6.5(9.3)$ & $8.0(7.3)$ & 0.235 \\
$\begin{array}{c}\text { IQR } \\
\text { Median duration } \\
\text { of nasogastric } \\
\text { decompression } \\
\text { (days) }+ \text { IQR }\end{array}$ & $2.0(3.0)$ & $3.0(1.3)$ & 0.334 \\
\hline
\end{tabular}

assigned or if conversion was required. Null hypotheses were tested two-sided and a $P$ value of 0.05 or less was considered statistical significant.

\section{Results}

Patients

A total of 109 patients were included in the trial based on a high suspicion of PPU (Fig. 1). Eight patients were 
Table 5 Postoperative pain

\begin{tabular}{llcc}
\hline & $\begin{array}{l}\text { Median VAS pain score } \\
\text { Laparoscopic repair }\end{array}$ & $\begin{array}{l}\text { Median VAS pain score } \\
\text { Open repair }\end{array}$ & $P$ value \\
\hline Day 1 + IQR & $3.8(3.0)$ & $5.15(2.5)$ & 0.001 \\
Day 3 + IQR & $2.1(2.5)$ & $3.0(2.4)$ & 0.035 \\
Day 7 + IQR & $1.0(2.0)$ & $1.85(2.8)$ & 0.036 \\
Day 28 + IQR & $0.3(0.7)$ & $0.0(1.7)$ & 0.748 \\
\hline
\end{tabular}

Table 6 Postoperative opiate usage

\begin{tabular}{llll}
\hline & $\begin{array}{l}\text { Opiate requirement } \\
\text { Laparoscopic repair }\end{array}$ & $\begin{array}{l}\text { Opiate requirement } \\
\text { Open repair }\end{array}$ & $P$ value \\
\hline Median duration (days) $+\mathrm{IQR}$ & $1.0(1.25)$ & $1.0(1.0)$ & 0.007 \\
Mean duration (days) $\pm \mathrm{SD}$ & $1.0 \pm 0.9$ & $1.6 \pm 0.9$ & 0.007 \\
\hline
\end{tabular}

Fig. 1 Patient flow chart

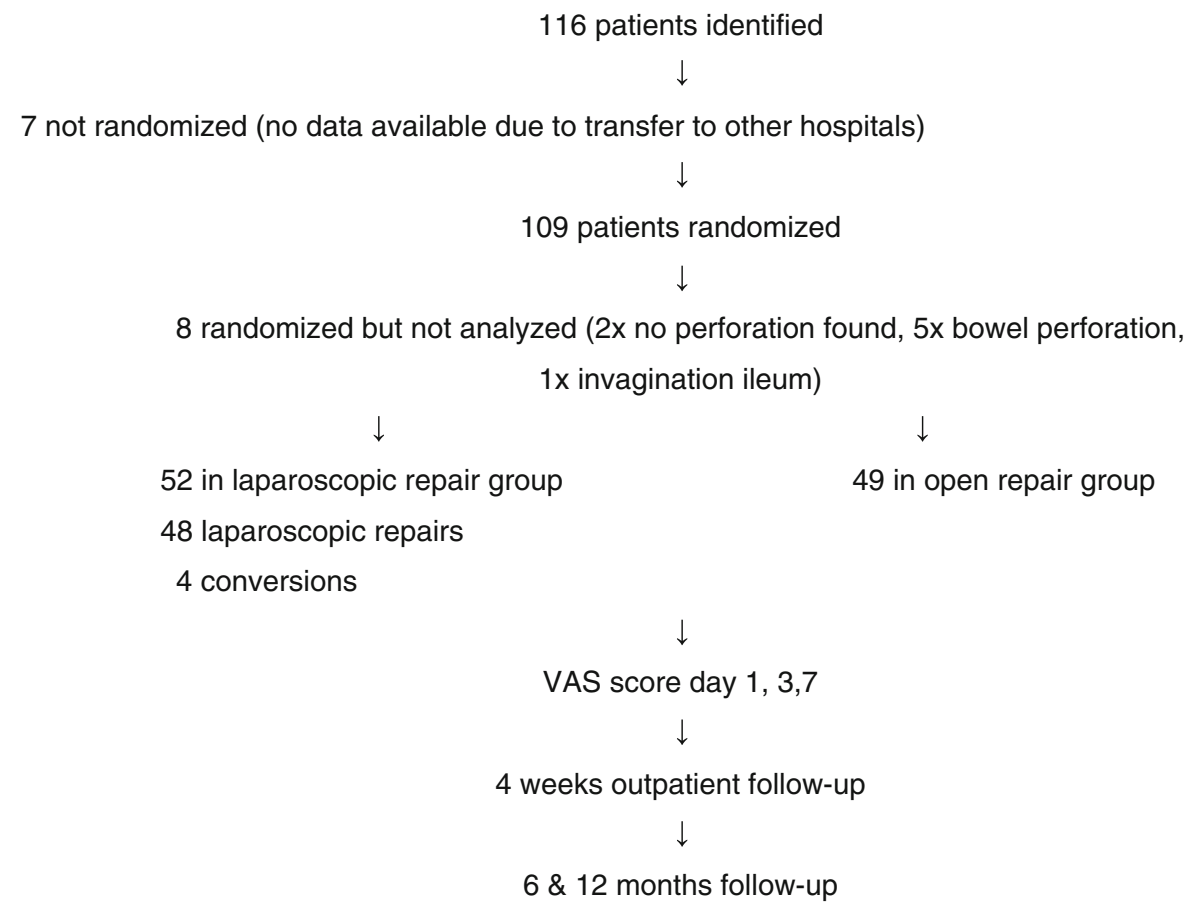

excluded during operation because no gastric perforation was detected or a defect in other parts of the digestive tract was uncovered. Data for these patients were not collected and the patients were excluded from further analysis. The remaining 101 patients made up the study population; their baseline parameters are given in Table 1. Fifty-two patients were randomized for laparoscopic repair and 49 for open repair of the perforation. Forty patients were female. The mean age of the patients was 61 years. The BMI (body mass index) was equally distributed between the groups, with a median of 22.5. Patients in both groups had been suffering from symptoms for a mean duration of $11 \mathrm{~h}$, and those in the laparoscopy group presented with a median Mannheim Peritonitis index [14] of 19.5, whereas those in the open group had a median Mannheim Peritonitis index of 16 .

Thirty patients reported the use of non-steroidal antiinflammatory drugs (NSAIDs; 17 laparoscopic versus 13 open), and 10 patients used proton pump inhibitors (6 laparoscopic versus 4 open). Patient history revealed gastric ulcer disease in 19 patients.

Intraoperative findings

The discovered ulcer perforations were found to have a mean diameter of $10 \mathrm{~mm}$, which did not differ between groups (Table 2). Location of the perforated ulcers was distributed equally between groups. Defects were located 
in the prepyloric region $(n=41)$, the postpyloric region $(n=34)$, and at the pylorus $(n=20)$. The median volume of lavage fluid used was 1,000 $\mathrm{ml}$ (range: $100-5,000 \mathrm{ml}$ ). The surgeon decided the amount of lavage used. There was no consensus on how much was necessary. Median blood loss did not differ between groups. Skin-to-skin time differed by $25 \mathrm{~min}$, favoring open repair of PPU (Table 2).

Intraoperative complications

Conversion to open surgery was required in four patients (8\%). Reasons for conversion included the inability to visualize the ulcer defect because of bleeding $(n=1 / 52$; $2 \%$ ), inability to reach the defect because of perforation in the vicinity of the gastroduodenal ligament and because of a dorsal gastric ulcer $(n=2 / 52 ; 4 \%)$, and inability to find the perforation $(n=1 / 52 ; 2 \%)$.

\section{Postoperative complications}

Complications were statistically equally distributed between the two groups (Table 3). There were $12 \mathrm{com}-$ plications in 9 patients in the laparoscopic group and 24 complications in 15 patients in the open group. Mortality was $4 \%$ in the laparoscopic group and $8 \%$ in the open group. In the laparoscopic group death was caused by sepsis due to leakage at the repair site. In the open group 3 patients died because of pulmonary problems (ARDS, pneumonia), and 1 patient died after complications following a cerebrovascular accident (CVA) combined with respiratory insufficiency.

\section{Discharge}

Time to discharge was similar for the two groups, with a median difference of 1.5 days (Table 4). Nasogastric decompression could be stopped after $2-3$ days in both groups (Table 4).

Pain

Visual analog pain scores were in favor of laparoscopic repair (Table 5; $p<0.005$ ). Although the median duration of opiate use in the two groups was 1.0, the mean duration in the open group was found to be 0.6 days longer than in the laparoscopic group (Table 6).

\section{VAS appearance of scar}

The VAS score for appearance of the scar left by the respective procedures (subjectively recorded in the same way as pain) differed by 2.3 points, favoring the laparoscopic procedure (7.7 vs. $5.4 ; P=0.033$ )

\section{Discussion}

The need for surgery for PPU has declined enormously in Europe and America with reported rates ranging from 50\% to $80 \%$, thanks to effective gastric acid-reducing medication [15]. For this reason, as well as because many surgeons prefer upper laparotomy, it took more time than expected to include 100 patients in our study. Reasons given by surgeons who prefer open repair were that it is a more familiar procedure and it can be completed faster than laparoscopy. It was also noted that patients often undergo operation at night, when the surgeon on call was not always laparoscopically trained.

Other randomized trials have already shown the feasibility of laparoscopic repair of PPU $[3,4,6,10]$. Only a few had more than 100 patients, and some emphasized results from subgroups of patients $[8,11,12]$. We did not subdivide our patients and included patients with risk factors for developing sepsis or conversion [10].

In eight of the original 109 patients (7\%) it became evident during the operation that the patient had a diagnosis different from PPU. In the patients who were randomized for laparoscopy this discovery revealed the benefit of laparoscopy as a diagnostic procedure indicating either an upper or lower abdominoplasty or continuation of the laparoscopy [16]. Conversion rate in the laparoscopy group was $8 \%(4 / 52)$. This is much lower than that reported in literature, where conversion rates as high as $60 \%$ were found $[3,4,6]$. This maybe partially explained by the fact that only trained and experienced laparoscopic surgeons (those performing at least 50 laparoscopic procedures a year) participated in this trial, confirming the belief that this procedure should only be done by experienced surgeons [3-5].

Operating time was significantly longer in the laparoscopy group (75 min versus $50 \mathrm{~min}$ ), which is comparable to reports in the literature $[3,10]$. A possible explanation for the longer operative time is that laparoscopic suturing is more demanding [9, 17], especially if the edges of the perforation are infiltrated and friable. Sutures easily tear out and it is more difficult to take large bites and to tie knots properly. Use of a single-stitch method described by Siu et al. [18], fibrin glue, or a patch might solve this problem [12, 19]. Another reason for the increase in operating time is the irrigation procedure. Irrigating through a 5-mm or even a $10-\mathrm{mm}$ trocar is time consuming, and suction of fluid decreases the volume of gas and reduces the pneumoperitoneum. There is no evidence that irrigation lowers the risk of sepsis [20], so it might only be necessary if there are food remnants in the abdomen; perhaps there is no need for it at all. One of the suspected risks of laparoscopic surgery is that of inducing sepsis by increasing bacterial translocation while establishing a 
pneumoperitoneum [6]. This danger could not be confirmed in our trial. Furthermore data suggest that there is a decrease in septic abdominal complications when laparosopic surgery is used [8].

Evidence already exists that laparoscopic correction of PPU causes less postoperative pain [6, 12, 17, 18]. The meta-analysis published by Lau [6] showed that eight out of ten studies showed a significant reduction in dosage of analgesics required in the laparoscopic group. Also, the three studies that had included VAS pain scores showed consistently lower pain scores, as was observed in our study as well. Whether this will lead to a better quality of life for patients, especially during the first weeks after surgery still needs to be analyzed. Although patients in our series who underwent laparoscopy had less postoperative pain, there was no difference in the length of hospital stay in our two groups. In fact, hospital stay overall in our patients was very long. This was most likely caused by the fact that many patients, especially the elderly, could not be discharged because of organizational reasons. Of the 101 patients, $41 \%$ were 70 years or older ( 24 in the laparoscopic group versus 17 in the open group). It appears that the age of PPU patients is increasing, and this will eventually represent a significant problem in the future $[2,3]$. One benefit of the laparoscopic procedure not often mentioned in literature [6] is cosmetic outcome. Nowadays patients are aware of this benefit, and sometimes this is the reason why they demand laparoscopic surgery.

In conclusion, the results of the LAMA trial confirm the results of other trials that laparoscopic correction of PPU is safe, feasible for the experienced laparoscopic surgeon, and causes less postoperative pain. Operating time was longer in the laparoscopic group and there was no difference in length of hospital stay or incidence of postoperative complications.

Acknowledgments The LAMA trial received a research grant from the Gastrostart organisation, the Netherlands. Financial support was provided by Johnson \& Johnson (Ethicon) Healthcare. Johnson \& Johnson did not have any involvement in the design and conduct of the study, the data analysis, or the interpretation of the results. The authors are grateful to Betty Hoogervorst and Hester Lingsma for their help with the statistics.

Open Access This article is distributed under the terms of the Creative Commons Attribution Noncommercial License which permits any noncommercial use, distribution, and reproduction in any medium, provided the original author(s) and source are credited.

\section{References}

1. Behrman SW (2005) Management of complicated peptic ulcer disease. Arch Surg 140:201-208

2. Svanes C (2000) Trends in perforated peptic ulcer: incidence, etiology, treatment, and prognosis. World J Surg 24:277-283

3. Lunevicius R, Morkevicius M (2005) Management strategies, early results, benefits and risk factors of laparoscopic repair of perforated peptic ulcer. World J Surg 29:1299-1310

4. Seelig MH, Seelig SK, Behr C et al (2003) Comparison between open and laparoscopic technique in the management of perforated gastroduodenal ulcers. J Clin Gastroenterol 3:226-229

5. Lunevicius R, Morkevicius M (2005) Risk factors influencing the early outcome results after laparoscopic repair of perforated duodenal ulcer and their predictive value. Langenbecks Arch Surg 390:413-420

6. Lau H (2004) Laparoscopic repair of perforated peptic ulcer: a meta-analysis. Surg Endosc 18:1013-1021

7. Mouret P, Francois Y, Vignal J et al (1990) Laparoscopic treatment of perforated peptic ulcer. Br J Surg 77:1006

8. Sanabria AE, Morales CH, Villegas MI (2005) Laparoscopic repair for perforated peptic ulcer disease. Cochrane Database Syst Rev (4):CD004778

9. Köhler L (1999) Endoscopic surgery: what has passed the test? World J Surg 23:816-824

10. Lunevicius R, Morkevicius M (2005) Comparison of laparoscopic vs open repair for perforated duodenal ulcers. Surg Endosc 19:1565-1571

11. Siu WT, Chau CH, Law BKB et al (2004) Routine use of laparoscopic repair for perforated peptic ulcer. Br J Surg 91:481-484

12. Lau WY, Leung KL, Kwong KH et al (1996) A randomized study comparing laparoscopic versus open repair of perforated peptic ulcer using suture or sutureless technique. Ann Surg 224:131-138

13. Nakajima S, Graham DY, Hattori T et al (2000) Strategy for treatment of Helicobacter infections in adults. II. Practical policy in 2000. Curr Pharm Des 15:1515-1529

14. Linder MM, Wacha H, Feldmann U et al (1987) Der Mannheimer peritonitis-index: ein instrument zur intraoperativen prognose der peritonitis. Chirurg 58:84-92

15. Sarosi GA, Jaiswal KR, Nwariaku FE et al (2005) Surgical therapy of peptic ulcers in the 21 st century: more common than you think. Am J Surg 190:775-779

16. Sauerland S, Agresta F, Bergamaschi R et al (2006) Laparoscopy for abdominal emergencies. Surg Endosc 20:14-29

17. Kirshtein B, Bayme M, Mayer $T$ et al (2005) Laparoscopic treatment of gastroduodenal perforations. Surg Endosc 19:14871490

18. Siu WT, Leong HT, Bonita KB et al (2002) Laparoscopic repair for perforated peptic ulcer. A randomized controlled trial. Ann Surg 235:313-319

19. Bertleff MJOE, Liem RSB, Bartels HL et al (2006) The Stamp method: a new treatment for perforated peptic ulcer? Surg Endosc 20:791-793

20. Schein M, Gecelter G, Freinkel W et al (1990) Peritoneal lavage in abdominal sepsis. A controlled clinical study. Arch Surg 125:1132-1135 